\title{
Numerical Analysis of Stratified and Slug Flows
}

\author{
Saliha Nouri, ${ }^{1,2}$ Zouhaier Hafsia $\mathbb{D}^{1,3}$ Salah Mahmoud Boulaaras $\mathbb{D}^{4,},{ }^{4,5}$ Ali Allahem $(\mathbb{D})$, \\ Salem Alkhalaf, ${ }^{7}$ and Baowei Feng ${ }^{8}$ \\ ${ }^{1}$ Department of Physics, College of Science and Arts at ArRass, Qassim University, Buraydah, Saudi Arabia \\ ${ }^{2}$ Department of Physics, University of Tunis, Tunis, Tunisia \\ ${ }^{3}$ Department of Physics, University of Tunis El-Manar, Tunis, Tunisia \\ ${ }^{4}$ Department of Mathematics, College of Science and Arts at ArRass, Qassim University, Buraydah, Saudi Arabia \\ ${ }^{5}$ Laboratory of Fundamental and Applied Mathematics of Oran (LMFAO), University of Oran 1, Oran 31000, Algeria \\ ${ }^{6}$ Department of Mathematics, College of Science, Qassim University, Buraydah, Saudi Arabia \\ ${ }^{7}$ Department of Computer Science, College of Science and Arts at ArRass, Qassim University, Buraydah, Saudi Arabia \\ ${ }^{8}$ Department Economic Mathematics, Southwestern University of Finance and Economics, Chengdu, China
}

Correspondence should be addressed to Zouhaier Hafsia; hafsia.zouhaier@gmail.com

Received 21 September 2021; Accepted 9 October 2021; Published 19 October 2021

Academic Editor: Sundarapandian Vaidyanathan

Copyright (C) 2021 Saliha Nouri et al. This is an open access article distributed under the Creative Commons Attribution License, which permits unrestricted use, distribution, and reproduction in any medium, provided the original work is properly cited.

\begin{abstract}
The main purpose of this study is to compare two-dimensional (2D) and three-dimensional (3D) two-phase models for both stratified and slug flows. These two flow regimes interest mainly the petroleum and chemical industries. The volume of fluid (VOF) approach is used to predict the interface between the two-phase flows. The stratified turbulent flow corresponds to the oil-water phases through a cylindrical pipe. To simulate the turbulent stratified flow, the $k-\omega$ turbulence model is used. The slug laminar flow concerns the kerosene-water phases through a rectangular microchannel. The simulated results are validated using the previous experimental results available in the literature. For the stratified flow, the axial velocity and the water volume fraction profiles obtained by $2 \mathrm{D}$ and 3D models approximate the measurement profiles at the same test section. Also, the T-junction in a $2 \mathrm{D}$ model affects only the inlet vicinity. For downstream, the 2D and 3D models lead to the same axial velocity and water volume distribution. For the slug flow, the simulated results show that the 3D model predicts the thin film wall contrary to the $2 \mathrm{D}$ model. Moreover, the 2D model underestimates the slug length.
\end{abstract}

\section{Introduction}

The stratified and the slug flows are particular two-phase phenomena existing in many applications such as petroleum transportation and chemical microreactors (see Desir et al. [1] and Santos et al. [2]). Using the slug flow, the transfer distance is reduced and the mixing process is enhanced along a microchannel. In the production pipelines, the pressure drop has a great effect on the operational costs and depends on the phase flow rates, the pipe diameter, and fluid properties (surface tension, density, and viscosity).

The two-phase flow can be divided into two kinds: the gas-liquid flow and the liquid-liquid flow. The gas-liquid flow regimes are the most studied regimes numerically and experimentally [3]. Since the pioneer experimental study of a liquid-liquid flow regime conducted by Charles et al. in [4], many other investigations have been conducted. Following the experimental investigation of Angeli and Hewitt in [5], different flow characteristics of two-phase immiscible fluids are measured including phase distribution and phase holdup of oil and water in horizontal pipe. Based on the experimental study conducted by Elseth in [6], the stratified flow occurs under low mixture velocity, and when the mixture velocity increases, the dispersed regime is observed. The contribution of the wall film associated with the slug flow on the mass transfer was demonstrated experimentally by Arsenjuk et al. in [7]. The available analytical solution is limited to a particular flow pattern. In the literature, different 
formulations for the determination of the pressure drop are available for core-annular flow with a laminar core and turbulent annulus [8].

The recent advances in computational fluid dynamics (CFD) have led to various numerical studies of the complex unsteady two-phase flow, overcoming the lack of laboratory information in some operating conditions. Al-Jadidi in [9] investigated a three-dimensional (3D) two-phase model with a large eddy simulation (LES) to better reproduce the turbulent structure to simulate the heavy oil and water flow regimes. The primary annular flow is identified and distinguished into three configurations: sudden contraction or sudden expansion and horizontal channel orifice. Carlos in [10] developed a numerical model of oil and water flow in horizontal pipes in order to study the transition between semidispersed and fully dispersed flows. The T-junction geometry in 2D and 3D models is the most used method to investigate the CAF and stratified and slug flows [11, 12]. Senapati and Dewangan in [13] used a 2D T-junction model to study the stratified flow with three different approaches to capture the interface between the oil and water phase. The coupled level set and volume of fluid (CLSVOF) can better reproduce the flow characteristics. For all these simulations, the $k-\omega$ turbulence model was adopted. 3D Reynolds Average Navier-Stokes (RANS) equations combined with the VOF method were used by Al-Yaari and Abu-Sharkh in [14] to simulate the oil and water stratified flow. The oil enters perpendicular to the water inlet along a $\mathrm{T}$-junction configuration. After a comparative study between the different $k-\varepsilon$ turbulence variants, the re-normalization group (RNG) $k-\varepsilon$ is chosen. The pressure drop associated to the slug flow was investigated experimentally by Kashid and Agar in [15] using a Y-junction as inlet configuration of the oil and water fluids. Desamala et al. in [16] conducted an experimental study to identify the transition of various flow regimes: plug to slug flow, slug to stratified wavy flow, and stratified wavy to stratified mixed flow. Based on $2 \mathrm{D} \mathrm{T}$ junction inlet configuration, the different transition flow patterns were validated. The RANS coupled with the VOF model and the $k-\varepsilon$ turbulence model were used.

The majority of numerical studies focused on the effects of the mixture velocity, the water-cut, the fluid properties, and the wetting properties on the flow regime. The two and three-dimensional flow nature is rarely analyzed. In this study, the numerical results of $2 \mathrm{D}$ and $3 \mathrm{D}$ models are compared for both turbulent stratified and laminar slug flows. This comparative study permits to identify the effect of the lateral dimension on the prediction of the interface interaction for oil-water stratified flow and kerosene-water slug flows.

\section{Problem Description}

In order to validate and examine the stratified and slug flows in $2 \mathrm{D}$ and $3 \mathrm{D}$, we consider the following geometric configurations previously studied experimentally. Figure 1 illustrates the $\mathrm{T}$-junction adopted to study the water and oil stratified flow regime using a $2 \mathrm{D}$ model. The flow direction is along the $z$-axis. The diameter of the pipe is the same as in the experimental study conducted by Elseth in [6] and is equal to $D=0.05575 \mathrm{~m}$. The length of the main pipe is $L_{1}=5 \mathrm{~m}$, and the branch pipe dimensions are as follows: $D_{1}=0.76 \mathrm{D}, \quad B_{1}=2.26 \mathrm{D}, B_{2}=0.72 \mathrm{D}$, and $B_{3}=1.45 \mathrm{D}$. The stratified flow is simulated for the mixture velocity $U_{m}=0.68 \mathrm{~m} / \mathrm{s}$ and the water-cut $C_{w}=0.5$, which corresponds to the following inlet velocities of water and oil imposed, respectively, at boundaries (1) and (2): $U_{w \text {,in }}=U_{o \text {,in }}=\left(U_{m} / 2\right) \times\left(D / D_{1}\right)=0.45 \mathrm{~m} / \mathrm{s}$. In the present $3 \mathrm{D}$ model, we suppose that the stratified flow is axisymmetric. Hence, the computational domain is composed of only the half cylinder as shown in Figure 2.

The geometric configuration of $\mathrm{T}$-junction to simulate the $2 \mathrm{D}$ slug flow is illustrated in Figure 3 . The same configuration was investigated experimentally by Cherlo et al. in [17]. The main and the branch microchannels meet at a right angle. The diameter of the microchannel is $D=590 \mu \mathrm{m}$. The water and kerosene inlets have the following length: $B_{4}=5 \mathrm{D}$. The length of the main channel $L=17 \mathrm{D}$. For the $3 \mathrm{D}$ slug flow model, the dimension of the rectangular pipe in the $x$-direction is equal to $h=500 \mu \mathrm{m}$. The inlet for water and kerosene at boundaries (1) and (2) is $U_{w, \text { in }}=U_{k, \text { in }}=10 \mathrm{ml} / \mathrm{h}$.

\section{Mathematical Model}

3.1. Governing Transport Equations. For turbulent flow, the transport equations for the two immiscible phases are the Reynolds Average Navier-Stokes (RANS) equations written in terms of the mixture properties. These equations concern the average mass conservation equation and the average momentum conservation equations and can be written in the following form [11]:

$$
\begin{aligned}
& \frac{\partial \rho}{\partial t}+\frac{\partial}{\partial x_{j}}\left(\rho U_{j}\right)=0, \quad j=1,2,3 \\
& \frac{\partial}{\partial t}\left(\rho U_{i}\right)+\frac{\partial}{\partial x_{j}}\left(\rho U_{j} U_{i}\right)=-\frac{\partial P}{\partial x_{i}}+\frac{\partial}{\partial x_{j}}\left[\left(\mu+\mu_{t}\right) \frac{\partial U_{i}}{\partial x_{j}}\right]+g_{i}+F_{i}, \quad i, j=1,2,3,
\end{aligned}
$$

where $\rho$ is the density of the mixture and $\mu$ is the dynamic molecular viscosity of the mixture defined by 


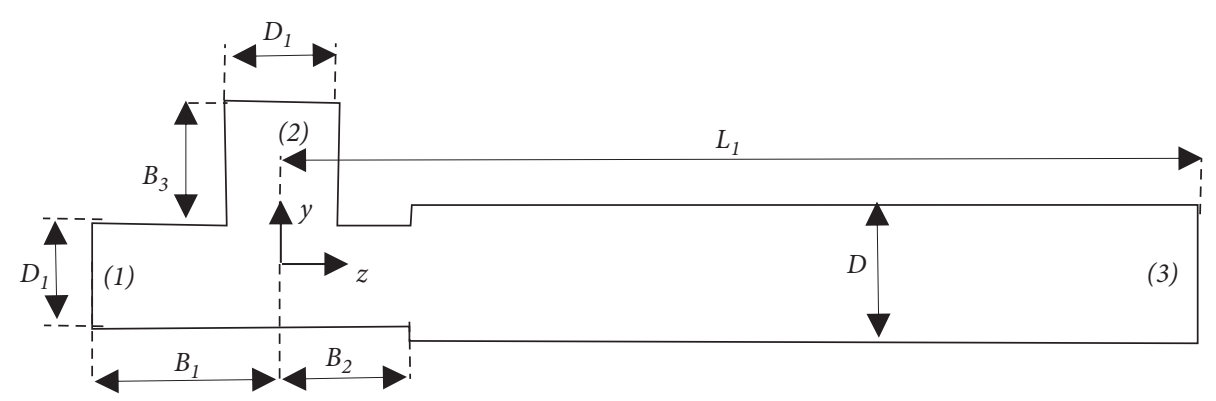

FIGURE 1: Sketch of 2D geometry of the T-junction adopted to simulate the stratified flow.

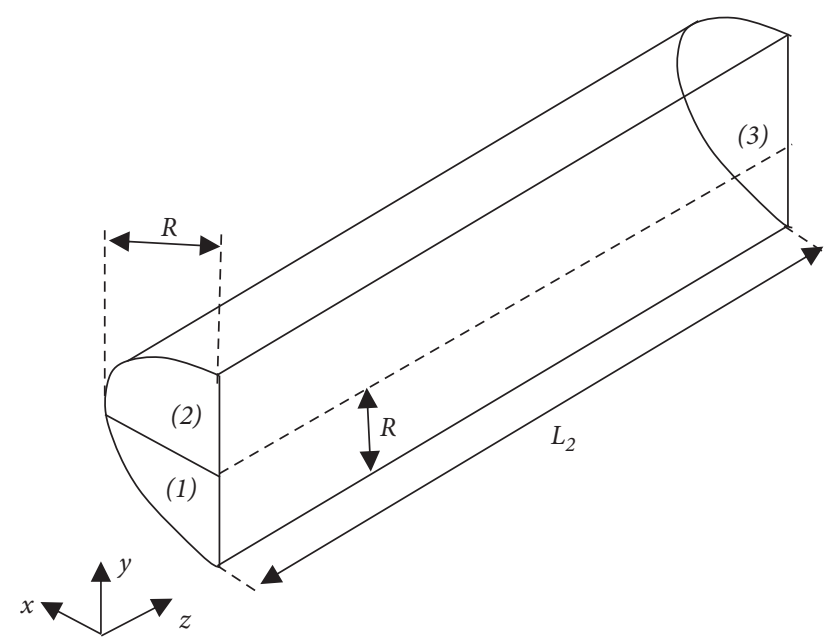

FIgURE 2: Sketch of $3 \mathrm{D}$ half cylinder adopted to simulate the stratified flow.

$$
\begin{aligned}
& \rho=\rho_{1} \alpha_{1}+\rho_{2} \alpha_{2}, \\
& \mu=\mu_{1} \alpha_{1}+\mu_{2} \alpha_{2},
\end{aligned}
$$

where $\rho_{1}$ and $\rho_{2}$ are, respectively, the densities of phases 1 and $2 ; \mu_{1}$ and $\mu_{2}$ are, respectively, the dynamic viscosities of phases 1 and $2 ; \alpha_{1}$ and $\alpha_{2}$ are the volume fractions of each phase such that $\alpha_{1}+\alpha_{2}=1 ; U_{i}$ are the mean velocity components; $P$ is the mean pressure; $\mu_{t}$ is the dynamic turbulent viscosity ( $\mu_{t}=0$ for the laminar slug flow); $g_{i}$ is the gravity acceleration field; and $F_{i}$ are the components of external force per unit volume associated to the interfacial tension.

Depending on the flow configuration, the better closure turbulent model can be chosen. In the present study, the $k-\omega$ model with shear stress transport (SST) is adopted as indicated by Shi et al. in [11]. Following this model, the turbulent viscosity $\mu_{t}$ is defined as follows [18]:

$$
\mu_{t}=f_{\mu} \frac{\rho k}{\omega} .
$$

Two added transport equations are needed to compute $\mu_{t}$ : for the turbulent kinetic energy, $k$, and for the specific dissipation rate, $\omega$ :

$$
\begin{aligned}
& \frac{\partial}{\partial t}(\rho k)+\frac{\partial}{\partial x_{j}}\left(\rho k U_{j}\right)=\frac{\partial}{\partial x_{j}}\left[\left(\mu+\frac{\mu_{t}}{\sigma_{k}}\right) \frac{\partial k}{\partial x_{j}}\right]+\rho\left(P_{k}-f_{2} \varepsilon\right) \\
& \frac{\partial}{\partial t}(\rho \omega)+\frac{\partial}{\partial x_{j}}\left(\rho \omega U_{j}\right)=\frac{\partial}{\partial x_{j}}\left[\left(\mu+\frac{\mu_{t}}{\sigma_{\omega}}\right) \frac{\partial \omega}{\partial x_{j}}\right]+\rho \omega\left(f_{1} C_{1 \omega} \frac{P_{k}}{k}-C_{2 \omega} \omega\right)
\end{aligned}
$$

where $P_{k}$ is the volumetric production rate of $k$ :

$$
P_{k}=\frac{\mu_{t}}{\rho}\left(\frac{\partial U_{i}}{\partial x_{j}}+\frac{\partial U_{j}}{\partial x_{i}}\right) \frac{\partial U_{i}}{\partial x_{j}},
$$

where $f_{\mu}, f_{1}$, and $f_{2}$ are low Reynolds damping functions.

The turbulent dissipation rate is given by

$$
\varepsilon=C_{D} \omega k \text {. }
$$

The following constants are commonly used:

$$
\begin{aligned}
\sigma_{k} & =2.0, \\
\sigma_{\omega} & =2, \\
C_{D} & =0.09, \\
C_{1 \omega} & =\frac{5}{9}, \\
C_{2 \omega} & =\frac{3}{40} .
\end{aligned}
$$

At high Reynolds numbers, the damping functions are set to unity and given by 


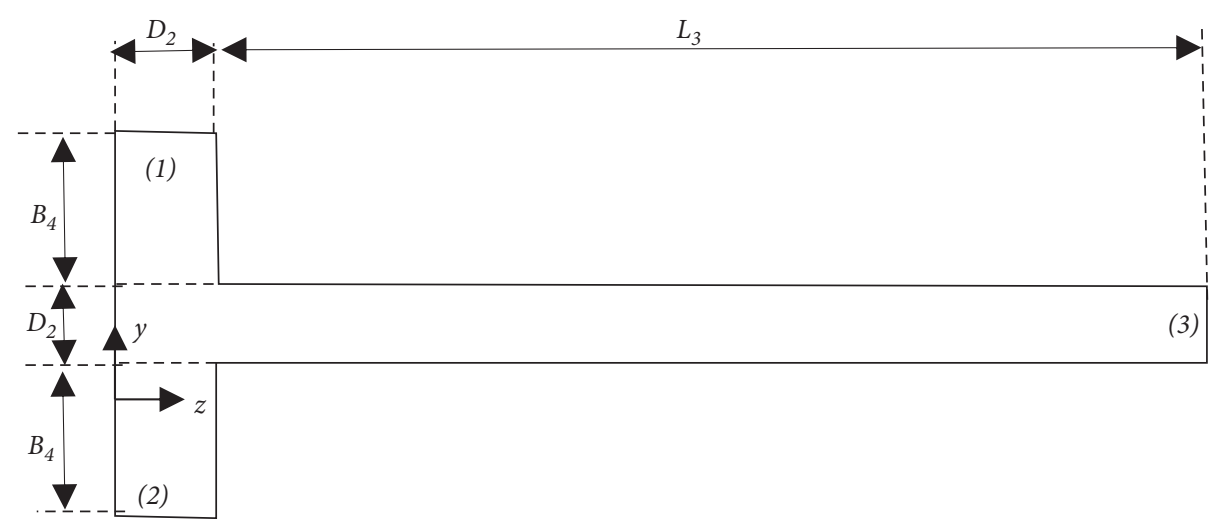

Figure 3: Side view of the computational domain adopted to simulate the slug flow.

$$
\begin{aligned}
& f_{\mu}=\frac{1 / 40+R_{e, t} / R_{k}}{1+R_{e, t} / R_{k}}, \quad R_{k}=6.0, \\
& f_{1}=\frac{1}{f_{\mu}} \frac{0.1+R_{e, t} / R_{\omega}}{1+R_{e, t} / R_{\omega}}, \quad R_{\omega}=2.7, \\
& f_{2}=\frac{5 / 18+\left(R_{e, t} / R_{B}\right)^{4}}{1+\left(R_{e, t} / R_{B}\right)^{4}}, \quad R_{B}=8.0,
\end{aligned}
$$

where $R_{e, t}$ is the turbulent Reynolds number defined as

$$
R_{e, t}=\frac{\rho k}{\mu \omega} .
$$

At high Reynolds numbers, the damping functions $f_{\mu}$, $f_{1}$, and $f_{2}$ tend to unity.

The surface tension at the interface between the two phases (the oil-water for stratified flow and the kerosenewater for the slug flow) can be transformed to a continuum surface force (CSF) as indicated by Brackbill et al. [19]. Following this model, the surface tension force is proportional to the curvature $\kappa$ :

$$
\vec{F}=\sigma \kappa \frac{\rho \vec{\nabla} \alpha_{1}}{1 / 2\left(\rho_{1}+\rho_{2}\right)},
$$

where $\kappa$ is the interface curvature computed by the divergence of the unit normal as

$$
\kappa=\vec{\nabla} \cdot\left(\frac{\vec{n}}{\bar{n}}\right) .
$$

The surface normal is given by the gradient of the volume fraction of the first phase:

$$
\vec{n}=\vec{\nabla} \alpha_{1}
$$

For the stratified flow, investigated experimentally by Elseth in [6], the surface tension between the oil and water phases is $\sigma=0.043 \mathrm{~N} / \mathrm{m}$. For the slug flow, investigated experimentally by Cherlo et al. in [17], the surface tension between the kerosene and water phases is $\sigma=0.045 \mathrm{~N} / \mathrm{m}$ and the contact angle $\theta=1^{0}$. These flow regimes are investigated through an academic version of a computational fluid dynamics code. The following fluid properties are used in the simulations:

(a) For the stratified flow, the fluid density and dynamic viscosity of each phase are as follows.

$$
\begin{aligned}
& \text { The first phase (oil): } \rho_{1}=790 \mathrm{~kg} / \mathrm{m}^{3} \\
& \text { and } \mu_{1}=0.00164 \text { Pa.s. } \\
& \text { The second phase (water): } \rho_{2}=1000 \mathrm{~kg} / \mathrm{m}^{3} \\
& \text { and } \mu_{2}=0.00102 \text { Pa.s. }
\end{aligned}
$$

(b) For the slug flow, the fluid density and dynamic viscosity of each phase are as follows.

$$
\begin{aligned}
& \text { The first phase (kerosene): } \rho_{1}=780 \mathrm{~kg} / \mathrm{m}^{3} \\
& \text { and } \mu_{1}=0.001 \text { Pa.s. } \\
& \text { The second phase (water): } \rho_{2}=998 \mathrm{~kg} / \mathrm{m}^{3} \\
& \text { and } \mu_{2}=0.001 \mathrm{~Pa} \text {.s. }
\end{aligned}
$$

3.2. Initial and Boundary Conditions. For the stratified flow, the horizontal pipe is filled with water at the initial time of simulation $(t=0 \mathrm{~s})$. In the case of the slug flow, the rectangular cross section channel is filled with the kerosene phase. For all simulated cases, at the pipe wall, a no-slip boundary condition is considered. At the outlet, denoted as (3) as indicated in Figures 1-3, zero pressure is specified. For the turbulent stratified flow, the turbulent kinetic energy and the specific dissipation rate at the inlet are calculated by the following equations:

$$
\begin{aligned}
& k=(I U)^{2}, \\
& \omega=\frac{\varepsilon}{C_{D} k}, \\
& \varepsilon=\frac{C_{D}^{3 / 4} k^{3 / 2}}{0.1 H},
\end{aligned}
$$

where $H$ is a characteristic inlet dimension as the hydraulic radius.

3.3. Numerical Methods. For the stratified flow, the computational domain contains 17,640 cells for 2D geometry and 495,000 cells for the half-cylinder geometry. The first cell layer in the cross section of the pipe is located at $0.2 \mathrm{~mm}$ in 


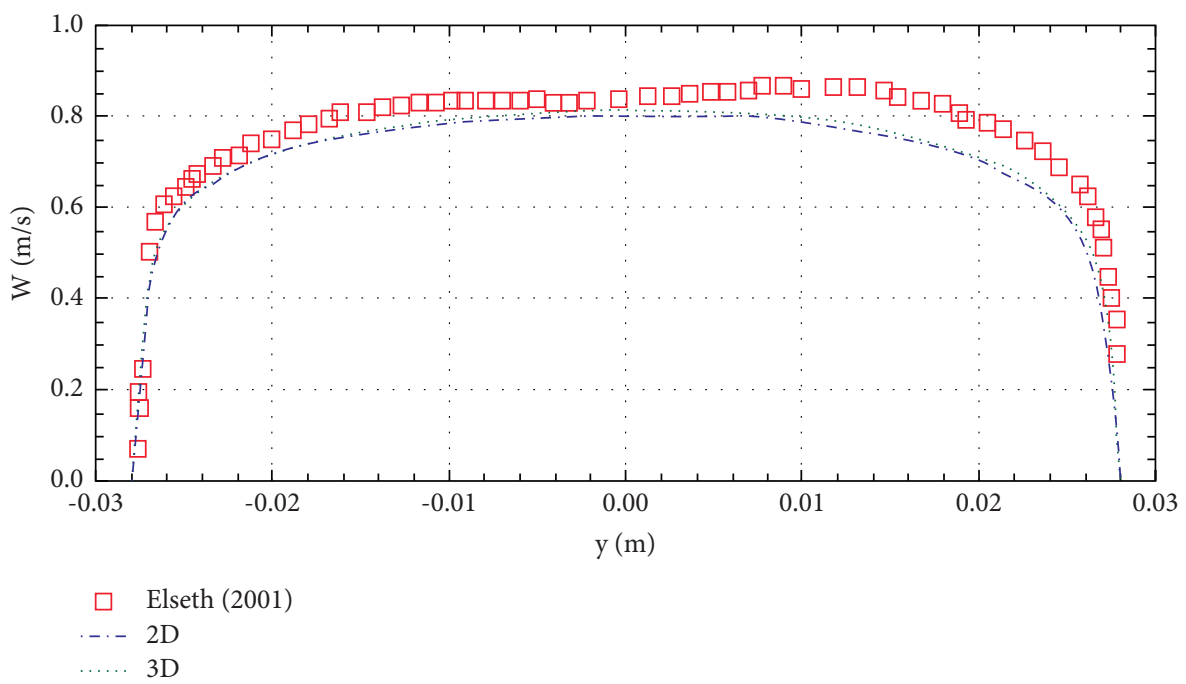

FIGURE 4: Comparison between the simulated axial velocity profile and the experimental results conducted by Elseth in [6] for a stratified flow.

order to satisfy that $y^{+}<5$. For the laminar slug flow, a local grid refinement is needed to well predict the velocity gradient near the wall. The grid numbers are 5100 and 71,400, respectively, for $2 \mathrm{D}$ and $3 \mathrm{D} \mathrm{T}$-junction geometries. The velocity-pressure coupling is solved by the PISO algorithm (pressure implicit with splitting of operator). For all solved variables, the residual is fixed to $10^{-5}$. The geometric reconstruction (piecewise-linear) method is chosen to accurately capture the interface variation between the two immiscible phases. The same method was adopted by Shi et al. in [20]. A variable time step is chosen with initial time step $\Delta t=10^{-5}$ s. For all simulated cases, the fully developed flow (FDF) is attained if the maximum axial velocity is unchanged with time.

\section{Validation of the Numerical Results}

4.1. Stratified Flow. The main differences between stratigraphic simulations by 2D and 3D models will be analyzed in terms of axial mixture velocity and water volume fraction. The simulated 2D stratified flow characteristics are compared to the experimental results of Elseth in [6] when the fully developed flow is attained. The axial velocities at the pipe axis and at $z=72 \mathrm{D}=4 \mathrm{~m}$ are represented in Figure 4 . There is a satisfactory agreement, but the faster flow in the oil region is not well reproduced. Figure 5 shows the predicted and measured water volume fractions. The sharp interface is correctly simulated. However, the existing waves at the oil and water interface cannot be predicted by the VOF model. To compare the effect of the inlet configuration, Figure 6 presents the water volume fraction in the axial plane of the pipe. The effect of the T-junction in the $2 \mathrm{D}$ model is only limited to the vicinity of the junction. The recirculation zone induced by the junction and by the expansion creates a water droplet having a dimension around twice the pipe diameter. There is no difference between these profiles far downstream.
4.2. Slug Flow. The slug flow is validated using the time evolution water volume distribution and the static pressure profiles for the $2 \mathrm{D}$ and $3 \mathrm{D}$ models. As confirmed by Cherlo et al. in [17], the mechanism of the slug formation can be explained through the following three stages (Figures 7(a)-7(e)).

(a) The water phase enters into the top microchannel: for the 2D model, this stage continues until the water stream blocks almost the entire cross section of the main channel (Figures 7(a), 7(b), 7(f), and 7(g)). For the $3 \mathrm{D}$ model, the water phase is separated from the wall at the junction (Figure $7(\mathrm{~g})$ ) and a thinner water interface at the main channel.

(b) For the $2 \mathrm{D}$ model, the water phase progresses in the main channel with a continuous oil entering from the low inlet channel. The shear stress and the pressure gradients exerted by the oil phase distorted the water stream at the end of the water inlet channel. A thin water layer connecting the two water streams is formed. The oil phase flows through the space between the wall and the water phase with high velocity (Figure $7(\mathrm{c})$ ). For the $3 \mathrm{D}$ model, the water slug breaks up before the instant $t=0.50 \mathrm{~s}$ and a thin kerosene layer adjacent to the wall is observed contrary to the $2 \mathrm{D}$ model.

(c) The thickness of the water layer is extremely reduced and breaks up to form a separate slug along the main channel (Figures 7(d) and 7(i)).

The formed slug progresses downstream, and a new droplet is formed when the break-up of the water layer at the junction occurs (Figures $7(\mathrm{e})$ and $7(\mathrm{j})$ ). Experimentally, the slug length in the same operating conditions taken in the present simulation is equal to $L_{s}=2.5 \mathrm{~mm}$. The length, $L_{s}$, is the measured distance between the upstream and downstream points along the plug interface at the developed regime flow. The calculated slug lengths are $2.0 \mathrm{~mm}$ and 


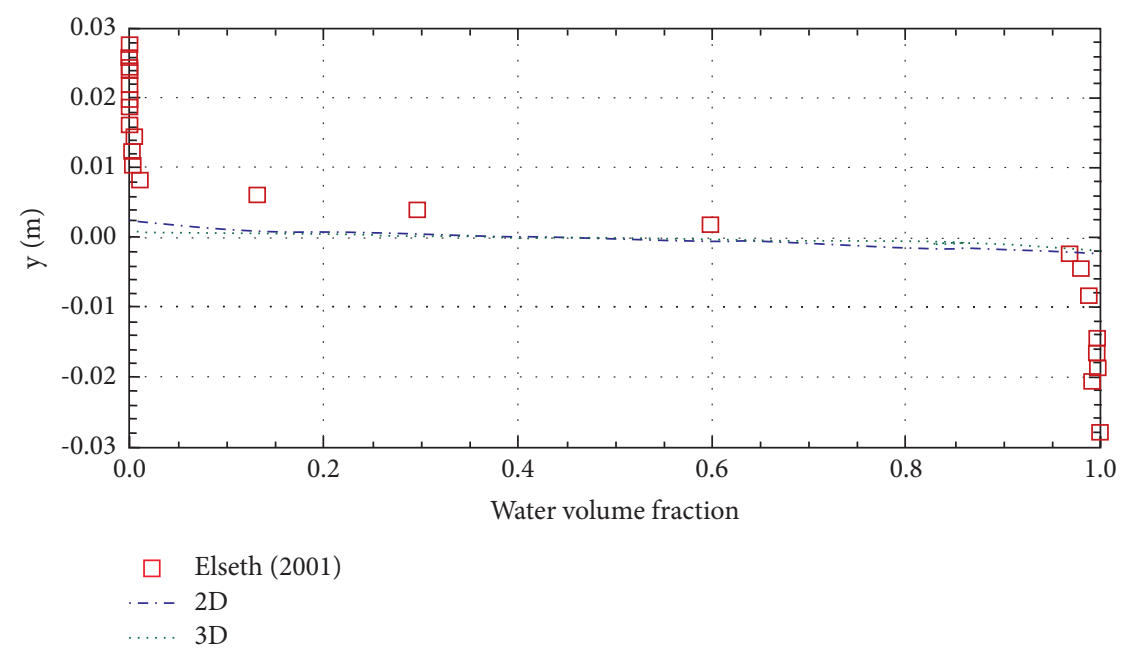

Figure 5: Comparison between the simulated water volume fraction profile and the experimental results conducted by Elseth in [6] for a stratified flow.

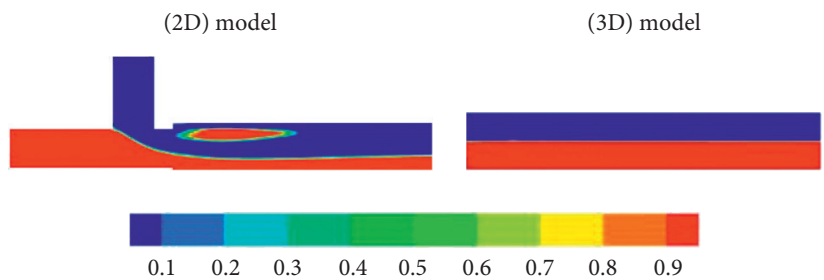

FIGURE 6: Water volume fraction at $y z$-plane simulated by $2 \mathrm{D}$ and 3D models for the fully developed stratified flow.

$2.2 \mathrm{~mm}$, respectively, for $2 \mathrm{D}$ and $3 \mathrm{D}$ models. It seems that the $2 \mathrm{D}$ model underestimates the slug length.

In order to validate the pressure drop in the $3 \mathrm{D}$ model, the computed static pressure will be compared to the Young-Laplace value. The variation of the static pressure along the axis of the microchannels at the instant $t=0.80 \mathrm{~s}$ is represented in Figure 8. The computed static pressure is higher in the dispersed phase (water) compared to the continuous phase (oil), and the increase of the pressure at the junction is due to the decrease of the velocity. The simulated pressure drop is around $310 \mathrm{~Pa}$ which approximates the Young-Laplace value calculated by the following equation:

$$
\Delta p=\frac{2 \sigma \cos \theta}{R_{2}}=305 \mathrm{~Pa}
$$

where $\sigma$ is the surface tension $(\sigma=0.045 \mathrm{~N} / \mathrm{m}) ; \theta$ is the contact angle $\left(\theta=1^{\circ}\right)$; and $R$ is the radius of the curvature equal to half width of the channel $\left(R_{2}=D_{2} / 2=0.295 \mathrm{~mm}\right)$.

The $3 \mathrm{D}$ model takes into account the two radii of curvature. Hence, the pressure drop for this model is approximately twice the simulated value for the $2 \mathrm{D}$ model which is around $147 \mathrm{~Pa}$ (Figure 8). In the latest model, only one radius of curvature contributes to the pressure drop and the second radius is infinity.

To show the effect of the surface tension on the slug regime, we consider the extreme case where $\sigma=0 \mathrm{~N} / \mathrm{m}$. Figure 9 illustrates the water volume fraction at two different instants simulated by the $2 \mathrm{D}$ model. Compared to the simulated case shown in Figure $7(\mathrm{e})$, the slug regime disappeared. 
(2D) model

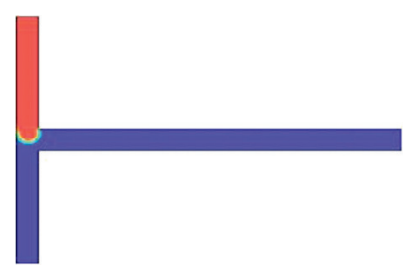

(a)

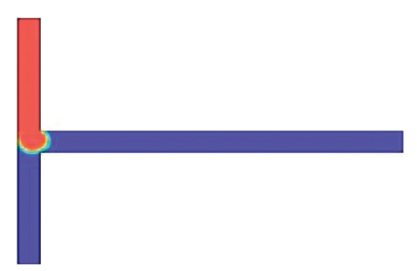

(b)

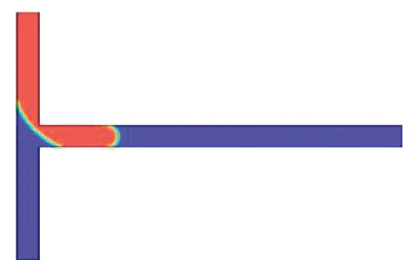

(c)

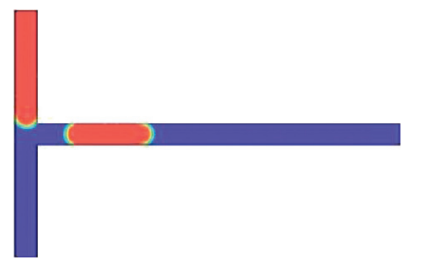

(d)

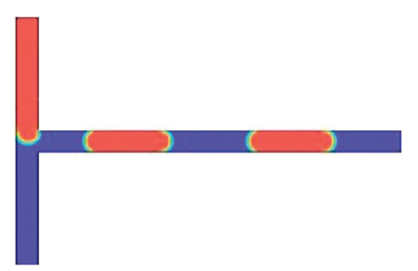

(e)
(3D) model

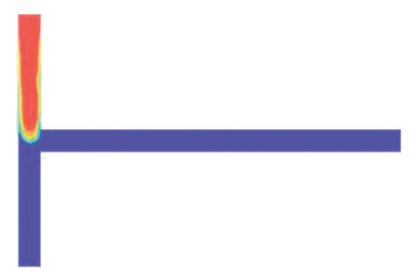

(f)

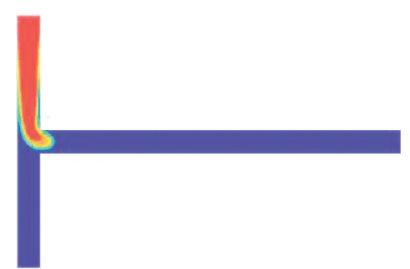

(g)

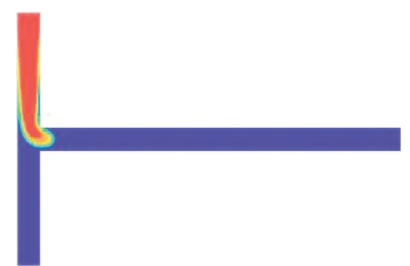

(h)

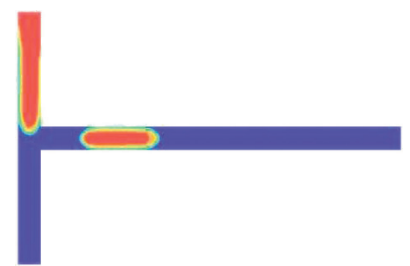

(i)

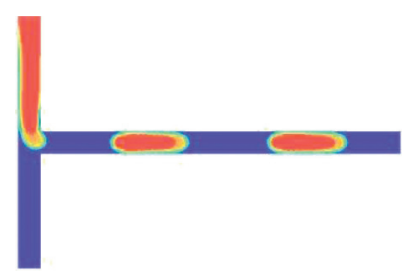

(j)

\section{$\begin{array}{lllllllll}0.1 & 0.2 & 0.3 & 0.4 & 0.5 & 0.6 & 0.7 & 0.8 & 0.9\end{array}$}

Figure 7: Water volume fraction simulated by 2D and 3D models at different times for the slug flow $\sigma=0.045 \mathrm{~N} / \mathrm{m}$. (a) $t=0.35 \mathrm{~s}$. (b) $t=0.39$ s. (c) $t=0.50$ s. (d) $t=0.55$ s. (e) $t=0.80$ s. (f) $t=0.35 \mathrm{~s}$. (g) $t=0.39 \mathrm{~s}$. (h) $t=0.50 \mathrm{~s}$. (i) $t=0.55 \mathrm{~s}$. (j) $t=0.80 \mathrm{~s}$. 


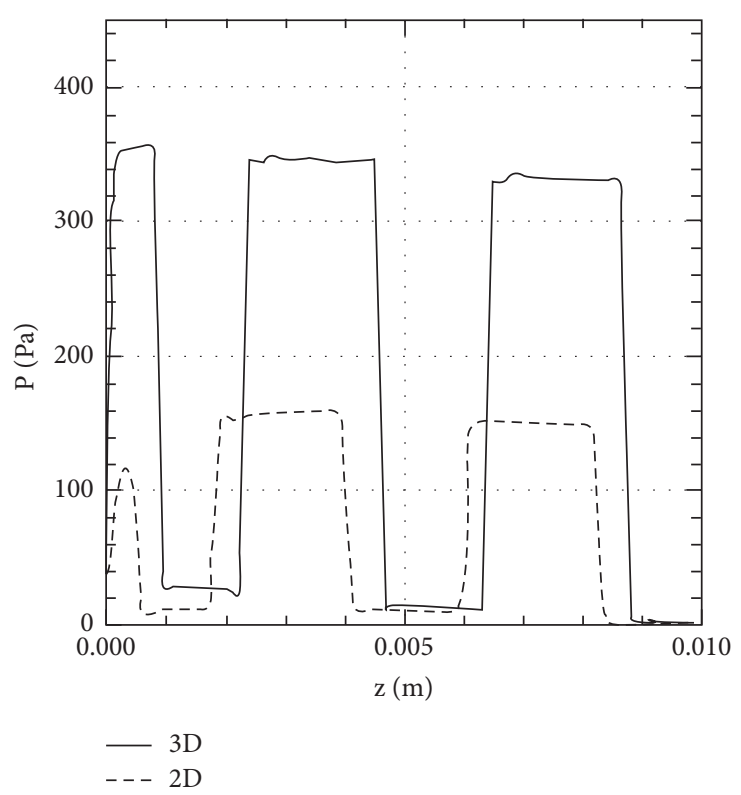

Figure 8: Pressure profile along the pipe length simulated by 2D and 3D models for the slug flow at $t=0.8 \mathrm{~s}$.
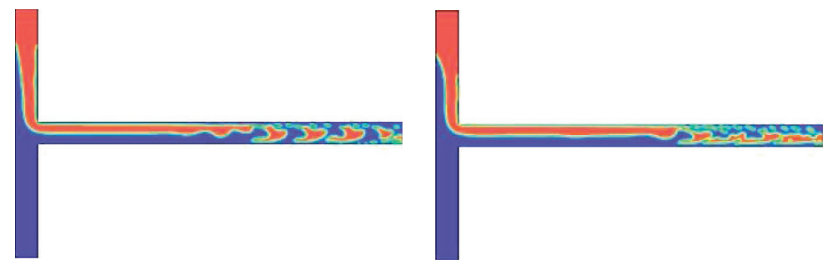

(a)

(b)

$\begin{array}{lllllllll}0.1 & 0.2 & 0.3 & 0.4 & 0.5 & 0.6 & 0.7 & 0.8 & 0.9\end{array}$

Figure 9: Water volume fraction simulated by $2 \mathrm{D}$ and $3 \mathrm{D}$ models at different times for the slug flow without surface tension. (a) $t=0.80 \mathrm{~s}$. (b) $t=1.19 \mathrm{~s}$.

\section{Conclusions and Perspectives}

The present two-phase flow model is based on RANS equations coupled with the VOF model and $k-\omega$ for turbulent flow. This model can predict the stratified flow characteristics by the $2 \mathrm{D}$ and $3 \mathrm{D}$ models. As expected, the surface tension has a crucial effect on the flow pattern. Without surface tension, the slug regime disappeared. The slug length obtained by the $2 \mathrm{D}$ model is slightly different from the $3 \mathrm{D}$ model; however, the thin film is not predicted by the $2 \mathrm{D}$ model.

As perspectives of this study, we can illustrate the following aspects:

(1) Investigation of the slug flow for multiple ducts.

(2) Development of a two-phase model taking into account the phase transfer.

(3) The effect of other inlet configurations such as $Y$ junction can be tested.
(4) The extension of this proposed model to study the effects of the air injection flow rate on the oil, water, and gas flow regimes and pressure drop through horizontal pipelines.

(5) Analysis of the effect of the microchannel inclination on the flow regimes.

\section{Data Availability}

No data were used in this study.

\section{Conflicts of Interest}

The authors declare that they have no conflicts of interest.

\section{Acknowledgments}

The authors gratefully acknowledge Qassim University, represented by the Deanship of Scientific Research, for the 
financial support (10133-cos-2020-1-3-I) during the academic year $1442 \mathrm{AH} / 2020 \mathrm{AD}$.

\section{References}

[1] P. Desir, T.-Y. Chen, M. Bracconi, B. Saha, M. Maestri, and D. G. Vlachos, "Experiments and computations of microfluidic liquid-liquid flow patterns," Reaction Chemistry and Engineering, vol. 5, no. 1, pp. 39-50, 2020.

[2] D. S. Santos, P. M. Faia, F. A. P. Garcia, and M. G. Rasteiro, "Oil/water stratified flow in a horizontal pipe: simulated and experimental studies using EIT," Journal of Petroleum Science and Engineering, vol. 174, pp. 1179-1193, 2019.

[3] S. Ban, W. Pao, and M. S. Nasif, "Numerical simulation of two-phase flow regime in horizontal pipeline and its validation," International Journal of Numerical Methods for Heat and Fluid Flow, vol. 28, no. 6, pp. 1279-1314, 2018.

[4] M. E. Charles, G. W. Govier, and G. W. Hodgson, "The horizontal pipeline flow of equal density oil-water mixtures," Canadian Journal of Chemical Engineering, vol. 39, no. 1, pp. 27-36, 1961.

[5] P. Angeli and G. F. Hewitt, "Flow structure in horizontal oilwater flow," International Journal of Multiphase Flow, vol. 26, no. 7, pp. 1117-1140, 2000.

[6] G. Elseth, An experimental study of oil/water flow in horizontal pipes, $\mathrm{PhD}$ thesis, Norwegian University of Science and Technology, Trondheim, Norway, 2001.

[7] L. Arsenjuk, F. Kaske, J. Franzke, and D. W. Agar, "Experimental investigation of wall film renewal in liquid-liquid slug flow," International Journal of Multiphase Flow, vol. 85, pp. 177-185, 2016.

[8] A. P. A. Kurban, Stratified liquid-liquid flow, $\mathrm{PhD}$ thesis, Imperial College of Science, London, UK, 1997.

[9] S. Al-Jadidi, Lubricated transport of heavy oil investigated by CFD, PhD Thesis, University of Leicester, Leicester, UK, 2017.

[10] F. T.-M. Carlos, Modeling of oil-water in horizontal and near horizontal pipes, $\mathrm{PhD}$ Thesis, University of Tulsa, Tulsa, OK, USA, 2006.

[11] J. Shi, M. Gourma, and H. Yeung, "CFD simulation of horizontal oil-water flow with matched density and medium viscosity ratio in different flow regimes," Journal of Petroleum Science and Engineering, vol. 151, pp. 373-383, 2017.

[12] Y. Zhao, G. Chen, and Q. Yuan, "Liquid-Liquid two-phase flow patterns in a rectangular microchannel," American Institute of Chemical Engineers, vol. 52, no. 12, pp. 4052-4060, 2006.

[13] S. K. Senapati and S. K. Dewangan, "Comparison of performance of different multiphase models in predicting stratified flow," Computational Thermal Sciences: International Journal, vol. 9, no. 6, pp. 529-539, 2017.

[14] M. A. Al-Yaari and B. F. Abu-Sharkh, "CFD prediction of stratified oil-water flow in a horizontal pipe," Asian Transactions on Engineering, vol. 1, no. 5, pp. 68-75, 2011.

[15] M. N. Kashid and D. W. Agar, "Hydrodynamics of liquidliquid slug flow capillary microreactor: flow regimes, slug size and pressure drop," Chemical Engineering Journal, vol. 131, no. 1-3, pp. 1-13, 2007.

[16] A. B. Desamala, V. Vijayan, A. Dasari, A. K. Dasmahapatra, and T. K. Mandal, "Prediction of oil-water flow patterns, radial distribution of volume fraction, pressure and velocity during separated flows in horizontal pipe," Journal of $\mathrm{Hy}$ drodynamics, vol. 28, no. 4, pp. 658-668, 2016.

[17] S. K. R. Cherlo, S. Kariveti, and S. Pushpavanam, "Experimental and numerical investigations of two-phase
(Liquid-Liquid) flow behavior in rectangular microchannels," Industrial \& Engineering Chemistry Research, vol. 49, no. 2, pp. 893-899, 2010.

[18] D. C. Wilcox, "Formulation of the $k-\omega$ turbulence model revisited," AIAA Journal, vol. 46, no. 11, pp. 2823-2838, 2008.

[19] J. U. Brackbill, D. B. Kothe, and C. Zemach, "A continuum method for modeling surface tension," Journal of Computational Physics, vol. 100, no. 2, pp. 335-354, 1992.

[20] J. Shi, A study on high-viscosity oil-water two-phase flow in horizontal pipes, $\mathrm{PhD}$ thesis, Cranfield University, Bedford, UK, 2015. 